OPEN ACCESS

Edited by:

Rita Raisman-Vozari, Paris Brain Institute-CNRS UMR 7225-Inserm U 1127-Sorbonne Université UM75, France

Reviewed by: Kun Xiong Independent Researcher, Central South University, China Rajkumar S. Kalra, Okinawa Institute of Science and Technology Graduate University, Japan

*Correspondence:

Nian-Sheng Tzeng

pierrens@mail.ndmctsgh.edu.tw

Received: 28 April 2021

Accepted: 13 July 2021

Published: 18 August 2021

Citation:

Ou H, Chien W-C, Chung $\mathrm{C}-\mathrm{H}$, Chang H-A, Kao Y-C, Wu P-C and

Tzeng N-S (2021) Association

Between Antibiotic Treatment of Chlamydia pneumoniae and Reduced

Risk of Alzheimer Dementia: A Nationwide Cohort Study in Taiwan. Front. Aging Neurosci. 13:701899

doi: 10.3389/fnagi.2021.701899

\section{Association Between Antibiotic Treatment of Chlamydia pneumoniae and Reduced Risk of Alzheimer Dementia: A Nationwide Cohort Study in Taiwan}

\author{
Hsun Ou ${ }^{1}$, Wu-Chien Chien ${ }^{2,3,4,5}$, Chi-Hsiang Chung ${ }^{2,3,5}$, Hsin-An Chang ${ }^{1,6}$, Yu-Chen Kao ${ }^{1,7}$, \\ Pei-Chuan $W^{1,8}$ and Nian-Sheng Tzeng ${ }^{1,6 *}$ \\ ${ }^{1}$ Department of Psychiatry, Tri-Service General Hospital, School of Medicine, National Defense Medical Center, Taipei, \\ Taiwan, ${ }^{2}$ Department of Medical Research, Tri-Service General Hospital, National Defense Medical Center, Taipei, Taiwan, \\ ${ }^{3}$ School of Public Health, National Defense Medical Center, Taipei, Taiwan, ${ }^{4}$ Graduate Institute of Life Sciences, National \\ Defense Medical Center, Taipei, Taiwan, ${ }^{5}$ Taiwanese Injury Prevention and Safety Promotion Association, Taipei, Taiwan, \\ ${ }^{6}$ Student Counseling Center, National Defense Medical Center, Taipei, Taiwan, ${ }^{7}$ Department of Psychiatry, Tri-Service \\ General Hospital, Song-Shan Branch, National Defense Medical Center, Taipei, Taiwan, ${ }^{8}$ Department of Psychiatry, Far \\ Eastern Memorial Hospital, New Taipei City, Taiwan
}

Background: Chlamydia pneumoniae $(\mathrm{CPn})$ is a common community-acquired pneumonia. In the literature, CPn infection is demonstrated to exhibit an association with Alzheimer dementia (AD). We executed the present nationwide, population-based research with the goal of probing the association of $\mathrm{CPn}$ infection and antibiotic therapy with $A D$ risk.

Methods: We conducted a cohort study using a database extracted from Taiwan's National Health Insurance Research Database (NHIRD). All medical conditions for each enrolled individuals were categorized using the International Classification of Diseases, ninth Revision classifications. Hazard ratios (HRs) and 95\% confidence intervals (Cls) for associations between CPn pneumonia-associated hospitalizations and AD were estimated using Fine and Gray's survival analysis and adjusted for comorbidities. The effects of the antibiotics on the HRs for AD in the patients with CPn pneumonia-associated hospitalization were also analyzed.

Results: Our analyses included 6,628 individuals, including 1,657 CPn-infected patients, as well as 4,971 controls matched by age, index date, and sex (1:3). In this study, patients hospitalized for CPn pneumonia exhibited a significantly higher AD risk (adjusted $\mathrm{HR}=1.599,95 \% \mathrm{Cl}=1.284-1.971, p<0.001$ ). We also noted an association of macrolide use ( $\geq 15$ days) and fluoroquinolone use ( $\geq 15$ days) with decreased AD risk.

Conclusions: We determined CPn pneumonia to be associated with a relatively high $A D$ risk. The result in this study confirmed the findings from previous literatures, by using a large, nationwide, population-based database. Appropriate macrolide and fluoroquinolone treatment may attenuate this risk.

Keywords: Chlamydia pneumoniae, Alzheimer dementia, National Health Insurance Research Database, macrolide, fluoroquinolone, nationwide cohort study 


\section{INTRODUCTION}

Dementia is one of the major global health problems. In Taiwan, the prevalence is $4-8 \%$ for a population aged $\geq 65$ in community studies (Sun et al., 2014), and it is therefore considered a heavy burden for the family, the caregivers, and society of these patients (Tzeng et al., 2015). Of the assorted varieties of dementia, Alzheimer dementia (AD) constitutes the most common, with an etiology that remains unclear; however, it is thought that the combined presence of amyloid and tau proteins and some genetic factors contribute to the pathogenesis of $\mathrm{AD}$.

Despite decades of research, much of the heritability of $\mathrm{AD}$ remains unexplained. Genes may play a role in some cases of AD. Most of the previous studies have indicated that the development of $\mathrm{AD}$ is not attributable to one or even a few mutations or gene polymorphisms. Instead, the disease genesis is considered multifactorial and may result in unknown environmental and host genetic factors (Balin et al., 2018).

Recently, research focused on the relationship between neuroinflammation and $\mathrm{AD}$. These studies indicated that inflammation would lead to neuronal damage and death in the brain. One study also demonstrated that neuroinflammation altered the expression and activity of amyloid precursor protein and promote amyloid formation (Al-Atrache et al., 2019). Additionally, microbial infections are believed to play a role in the development of neuroinflammation and increased the risk of $\mathrm{AD}$ (Boelen et al., 2007; Bloom, 2014; Al-Atrache et al., 2019).

Among various microbial infections, researchers have proposed Chlamydia pneumoniae $(\mathrm{CPn})$ may be implicated in $\mathrm{AD}$ pathology and certain antibiotics were determined to exhibit some degree of effectiveness in treating moderate $\mathrm{AD}$ (Loeb et al., 2004); however, the results of human and animal studies in past decades were inconsistent (Maheshwari and Eslick, 2015; Woods et al., 2020). Because nationwide population-based studies have yet to be executed to corroborate the CPn infection$\mathrm{AD}$ association. Accordingly, in our attempt to bridge this gap, we executed our current study by employing information gleaned from the National Health Insurance Research Database (NHIRD) of Taiwan. The aim of this study is to determine the association between $\mathrm{CPn}$ pneumonia, antibiotics therapy, and the risk of $\mathrm{AD}$.

\section{METHODS}

\section{Data Sources}

Data for our nationwide, population-based, cohort study were obtained from the inpatient care records and registration files from the NHIRD. The National Health Insurance (NHI) program

Abbreviations: AD, Alzheimer dementia; APOE, apolipoprotein E4; CI, confidence interval; CCI, Charlson comorbidity index; CAD, coronary artery disease; CNS, central nervous system; CPn, Chlamydia pneumoniae; DM, diabetes mellitus; DDD, defined daily dosage; LHID, longitudinal Health Insurance Database; NHIRD, National Health Insurance Research Database; NHI, National Health Insurance; HR, hazard ratio. was implemented in 1995 and provides healthcare coverage to 99\% of the population (being more than 23 million people). The NHI Administration randomly reviews the records of ambulatory care visits and in-patient claims periodically so as to verify the accuracy of the diagnoses (National Health Insurance Administration National Health Insurance Regulations). The details of the program have been documented in previous studies (Chang et al., 2018; Chao et al., 2018). It should be noted that some studies have demonstrated the accuracy and validity of several diagnoses in the NHIRD, including diabetes mellitus (DM), cancer, myocardial infarction, and the central nervous system diseases, such as Tourette syndrome, and stroke.

\section{Study Population}

This study involved a cohort design. Using the NHIRD, we selected adult patients aged $>50$ years who had been diagnosed with $\mathrm{AD}$ (331.0) based on the International Classification of Diseases, 9th Revision, Clinical Modification (ICD-9-CM) codes between 2000 and 2015, and confirmed these patients' diagnoses by linking the records of at least three visits for $\mathrm{AD}$ in consecutive years, or at least one hospitalization for $\mathrm{AD}$, during the study period. All diagnoses of $\mathrm{AD}$ were made by board-certified psychiatrists or neurologist in Taiwan. The date of the $\mathrm{AD}$ diagnosis was at least 1 year after the $\mathrm{CPn}$ pneumonia diagnosis. We identified patients with $\mathrm{CPn}$ pneumonia using the ICD9-CM code 483.1. We confirmed these patients' diagnoses by linking the records of at least one hospitalization for $\mathrm{CPn}$ pneumonia, in the study period, and the reason why we decided on the enrolled exposures of $\mathrm{CPn}$ as pneumonia due to $\mathrm{CPn}$, was because one previous population-based study had confirmed the accuracy of the diagnosis of pneumonia from the NHIRD as $98.3 \%$ (Su et al., 2014). For each patient with Cpn pneumonia included in our study, three controls were selected via 1:3 matching by age, sex, and the number of medical follow-ups $(\mathrm{N}=4971)$ in the NHIRD. All insurance claims were scrutinized by medical reimbursement specialists, and peer reviews were undertaken according to the standard and clinical diagnostic criteria.

\section{Covariates}

The covariates included sex, age groups $(50-64, \geq 65$ years), marital status, education ( $<12$ years, $\geq 12$ years), seasons, geographical area of residence (north, center, south, and east of Taiwan), urbanization level of residence (levels 1-4), and monthly income (in New Taiwan Dollars [NT\$]; $<18,000$, $18,000-34,999, \geq 35,000)$. The urbanization level of residence was defined according to the population and various indicators of the level of development. Level 1 was defined as a population of $>1,250,000$, and a specific designation as political, economic, cultural, and metropolitan development. Level 2 was defined as a population between 500,000 and 1,249,999, and as playing a key role in the politics, economy, and culture. Urbanization levels 3 and 4 were defined as a population between 149,999 and 499,999, and $<149,999$, respectively (Chang et al., 2014).

Data on the usage of macrolides and fluoroquinolones antibiotics were acquired from the Longitudinal Health 
Insurance Database (LHID), a sub-database of the NHIRD. The data of the defined daily dosage (DDD) were obtained from the WHO Collaborating Centre for Drug Statistics Methodology (https://www.whocc.no/), and the duration of the usage of antibiotics was calculated by dividing the cumulative dosages by the DDD of the antibiotics.

\section{Comorbidity}

The comorbidities, including DM (ICD-9-CM 250), hypertension (ICD-9-CM 401.1, 401.9, 402.10, 402.90, 404.10, 404.90, 405.1, and 405.9), hyperlipidemia (ICD-9-CM 272), coronary artery disease (CAD, ICD-9-CM code 410-414), obesity (ICD-9-CM 278), all cancers (ICD-9-CM 140-208), and other chlamydia infections (ICD-9-CM codes: 077.98, ICD-9-CM 078.88, 079.88, 079.98, 099.41, and 099.5) and other pneumonia (ICD-9-CM codes: 480-486, except 483.1). These comorbidities were included with the references from previous studies using health databases (Wright et al., 2015; Gottesman et al., 2017).

The Charlson comorbidity index (CCI) was employed to execute the assessment of the aforementioned comorbidities. In the CCI, ICD-9-CM codes are used as the basis for the establishment of categories for comorbidities, and each of the established categories is scored (van den Berg et al., 2013, 2014; Wong et al., 2014); to derive a single comorbidity score, all CCI scores are combined, with 0 indicating no comorbidities and higher scores $(1,2,3, \geq 4)$ indicating higher comorbidity burdens (Needham et al., 2005).

\section{Definitions of Patients With CPn Pneumonia}

Only patients diagnosed with CPn pneumonia of more than 1 year prior to the index date were considered. CPn pneumonia was identified from the NHIRD by using the corresponding ICD-9 code (ICD-9-CM code 483.1). All the covariates as aforementioned were included.

\section{Statistical Analysis}

Categorical variables, which were presented as percentages, were compared using the $\chi^{2}$ tests and the Fisher's exact test. Continuous variables, which were presented as the mean and $\mathrm{SD}$, were compared using the Student's $t$-tests. The primary goal of this study was to determine as to whether a patient's clinical characteristics, such as CPn pneumonia, were associated with $\mathrm{AD}$. Associations between those outcomes and clinical characteristics were investigated using the Fine and Gray's survival analysis in a generalized estimating equation (GEE) model. The regression results are presented as adjusted HRs with corresponding 95\% CIs. The threshold for statistical significance was $p<0.05$. All data analyses were conducted using the SPSS V.22 (SPSS).

\section{RESULTS}

\section{Enrolled Samples}

Supplementary Figure $\mathbf{1}$ is a flowchart of the patient enrollment procedure. From the NHIRD, we identified 1,657 patients who received a CPn pneumonia diagnosis during our defined study period (2000-2015); these patients were matched 1:3 with patients without $\mathrm{CPn}(\mathrm{N}=4,971)$ according to age, sex, number of visits to medical facilities, and comorbidities.

\section{Sample Characteristics}

In total, 1,657 patients were diagnosed with Cpn pneumonia during the study period. A total of 182 enrollee were identified with $\mathrm{AD}$ during the follow-up period.

Table 1 shows the sex, age, marital status and comorbidities of the patients with Cpn pneumonia. When compared with controls, the patients with Cpn pneumonia tended to have higher rate of hypertension, hyperlipidemia, other pneumonia, anxiety, sleep disorder and CCI scores.

Patients with CPn pneumonia also tended to be living in northern and middle Taiwan and residing more in the regions of urbanization levels 1 and 2. There were no differences in the distribution of sex, age, marital status, and education between these two groups.

\section{Hazard Ratios Analysis of AD in the Patients With CPn Pneumonia}

In our applied Fine \& Gray's competing risk model, compared with the control group, we determined patients with CPn pneumonia to have a higher risk of $\mathrm{AD}$ (adjusted hazard ratio $[\mathrm{HR}]=1.599,95 \% \mathrm{CI}=1.284-1.971, p<0.001$; Table 2) after we adjusted for urbanization level/geographic region, sex, marital status, education, comorbidities (including CCI scores), age, antibiotic use, and insurance premium.

In addition, we observed patients with hyperlipidemia, CAD, anxiety, sleep disorder, and alcohol use disorder to be at a relatively high risk of AD. Furthermore, we noted those receiving care from a medical center or regional hospital to exhibit a relatively high risk of $\mathrm{AD}$. Conversely, patients who sought medical care in Autumn and with cancer revealed a reduced risk of $\mathrm{AD}$. Although hypertension exhibited a significant difference in the patients with CPn pneumonia group at baseline (see in Table 1), it did not significantly affect developing AD by the logistic regression model ( $p=0.087$; Table 2$)$.

\section{Antibiotics and the Risk of AD in Patients With CPn Pneumonia}

Table 3 shows the usage of antibiotics in the risk of $\mathrm{AD}$ in patients with CPn pneumonia, and that the usage of macrolides $(\geq 15$ days) and fluoroquinolones ( $\geq 15$ days), were associated with a decreased risk of dementia.

\section{Kaplan-Meier Curves for the Cumulative Incidence of AD in Patients With CPn Pneumonia}

The cumulative incidences of $\mathrm{AD}$ were 931.01 per $10^{5}$ person-years and 764.22 per $10^{5}$ person-years, in the study cohort and comparison cohort group, respectively (Supplementary Table 1). The difference between the two groups was significant (log-rank test, $p<0.001$; Figure 1). After 8 years of tracking, the cumulative incidence of $\mathrm{AD}$ in patients 
TABLE 1 | Characteristics of study at the baseline.

\begin{tabular}{|c|c|c|c|c|c|}
\hline $\begin{array}{l}\text { Chlamydia pneumoniae } \\
\text { Variables }\end{array}$ & \multicolumn{2}{|c|}{ With } & \multicolumn{2}{|c|}{ Without } & $p$ \\
\hline Gender & & & & & 0.999 \\
\hline Male & 1,002 & 60.47 & 3,006 & 60.47 & \\
\hline Female & 655 & 39.53 & 1,965 & 39.53 & \\
\hline Age groups (years) & & & & & 0.999 \\
\hline $50-64$ & 809 & 48.82 & 2,427 & 48.82 & \\
\hline$\geq 65$ & 848 & 51.18 & 2,544 & 51.18 & \\
\hline Marital status & & & & & 0.681 \\
\hline Without & 809 & 48.82 & 2,456 & 49.41 & \\
\hline Insured premium (NT\$) & & & & & $<0.001$ \\
\hline$<18,000$ & 1,607 & 96.98 & 4,926 & 99.09 & \\
\hline $18,000-34,999$ & 41 & 2.47 & 43 & 0.87 & \\
\hline$\geq 35,000$ & 9 & 0.54 & 2 & 0.04 & \\
\hline Diabetes mellitus & 275 & 16.60 & 838 & 16.86 & 0.820 \\
\hline Hypertension & 416 & 25.11 & 988 & 19.88 & $<0.001$ \\
\hline Hyperlipidemia & 77 & 4.65 & 171 & 3.44 & 0.030 \\
\hline Coronary artery disease & 178 & 10.74 & 587 & 11.81 & 0.249 \\
\hline Obesity & 0 & 0.00 & 2 & 0.04 & 0.414 \\
\hline Cancer & 122 & 7.36 & 400 & 8.05 & 0.400 \\
\hline $\begin{array}{l}\text { Other Chlamydiae } \\
\text { infections or diseases }\end{array}$ & 1 & 0.06 & 0 & 0.00 & 0.250 \\
\hline CCl_R & & & & & $<0.001$ \\
\hline 0 & 1,067 & 64.39 & 3,199 & 64.35 & \\
\hline 1 & 440 & 26.55 & 1,126 & 22.65 & \\
\hline 2 & 105 & 6.34 & 414 & 8.33 & \\
\hline 3 & 28 & 1.69 & 153 & 3.08 & \\
\hline$\geq 4$ & 17 & 1.03 & 79 & 1.59 & \\
\hline Season & & & & & 0.172 \\
\hline Spring (Mar-May) & 453 & 27.34 & 1,287 & 25.89 & \\
\hline Summer (Jun-Aug) & 395 & 23.84 & 1,127 & 22.67 & \\
\hline Autumn (Sep-Nov) & 363 & 21.91 & 1,082 & 21.77 & \\
\hline Winter (Dec-Feb) & 446 & 26.92 & 1,475 & 29.67 & \\
\hline Location & & & & & $<0.001$ \\
\hline Northern Taiwan & 742 & 44.78 & 1,850 & 37.22 & \\
\hline Middle Taiwan & 458 & 27.64 & 1,413 & 28.42 & \\
\hline Southern Taiwan & 287 & 17.32 & 1,325 & 26.65 & \\
\hline
\end{tabular}


TABLE 1 | Continued

\begin{tabular}{|c|c|c|c|c|c|}
\hline \multirow{2}{*}{$\begin{array}{l}\text { Chlamydia pneumoniae } \\
\text { Variables }\end{array}$} & \multicolumn{2}{|c|}{ With } & \multicolumn{2}{|c|}{ Without } & \multirow[t]{2}{*}{$p$} \\
\hline & $\mathbf{n}$ & $\%$ & $\mathbf{n}$ & $\%$ & \\
\hline Eastern Taiwan & 167 & 10.08 & 352 & 7.08 & \\
\hline Outlets islands & 3 & 0.18 & 31 & 0.62 & \\
\hline Urbanization level & & & & & $<0.001$ \\
\hline 1 (The highest) & 570 & 34.40 & 1,549 & 31.16 & \\
\hline 2 & 844 & 50.94 & 2,188 & 44.02 & \\
\hline 3 & 84 & 5.07 & 346 & 6.96 & \\
\hline 4 (The lowest) & 159 & 9.60 & 888 & 17.86 & \\
\hline Level of care & & & & & $<0.001$ \\
\hline Hospital center & 591 & 35.67 & 1,542 & 31.02 & \\
\hline Regional hospital & 749 & 45.20 & 1,573 & 31.64 & \\
\hline Local hospital & 317 & 19.13 & 1,856 & 37.34 & \\
\hline
\end{tabular}

CCI_R: Charlson Comorbidity Index, dementia removed; P: Chi-square/Fisher exact test on category variables and t-test on continue variables.

TABLE 2 | Factors for Alzheimer dementia by using the analysis of Fine and Gray's competing risk model.

\begin{tabular}{|c|c|c|c|c|c|c|c|c|}
\hline \multirow[b]{2}{*}{ Variables } & \multicolumn{8}{|c|}{ Competing risk in the model } \\
\hline & Crude HR & $95 \% \mathrm{Cl}$ & $95 \% \mathrm{Cl}$ & $\boldsymbol{P}$ & Adjusted HR & $95 \% \mathrm{Cl}$ & $95 \% \mathrm{Cl}$ & $\boldsymbol{P}$ \\
\hline Chlamydia pneumonia (reference: without) & 1.676 & 1.425 & 1.902 & $<0.001$ & 1.599 & 1.284 & 1.971 & $<0.001$ \\
\hline Male (reference: female) & 1.182 & 1.007 & 1.387 & 0.041 & 1.164 & 0.989 & 1.369 & 0.067 \\
\hline Hyperlipidemia (reference: without) & 1.497 & 1.298 & 1.830 & $<0.001$ & 1.427 & 1.252 & 1.724 & 0.002 \\
\hline Coronary artery disease (reference: without) & 1.492 & 1.396 & 1.657 & $<0.001$ & 1.478 & 1.357 & 1.640 & $<0.001$ \\
\hline Hypertension (reference: without) & 0.843 & 0.713 & 1.007 & 0.064 & 1.060 & 0.723 & 1.222 & 0.087 \\
\hline Cancer (reference: without) & 0.376 & 0.265 & 0.535 & $<0.001$ & 0.400 & 0.280 & 0.572 & $<0.001$ \\
\hline Depression (reference: without) & 2.066 & 1.106 & 3.860 & 0.023 & 1.551 & 0.825 & 2.917 & 0.173 \\
\hline Anxiety (reference: without) & 1.627 & 1.333 & 1.901 & $<0.001$ & 1.638 & 1.394 & 1.926 & $<0.001$ \\
\hline Alcohol use disorder (reference: without) & 5.351 & 2.001 & 14.311 & 0.001 & 5.778 & 2.138 & 15.637 & 0.001 \\
\hline Sleep disorder (reference: without) & 2.090 & 1.180 & 3.701 & 0.011 & 2.011 & 1.129 & 3.584 & 0.018 \\
\hline CCl_R 1 (reference: CCl_R: 0) & 1.546 & 1.303 & 1.835 & $<0.001$ & 1.353 & 1.134 & 1.615 & 0.001 \\
\hline CCl_R 2 (reference: CCI_R: 0) & 1.348 & 1.036 & 1.745 & 0.026 & 1.081 & 0.826 & 1.415 & 0.599 \\
\hline CCl_R 3 (reference: CCI_R: 0) & 1.759 & 1.201 & 2.576 & 0.004 & 1.436 & 0.976 & 2.113 & 0.066 \\
\hline Autumn (reference: Spring) & 0.744 & 0.593 & 0.933 & 0.011 & 0.704 & 0.560 & 0.885 & 0.003 \\
\hline Medical center (reference: local hospital) & 1.689 & 1.570 & 1.832 & $<0.001$ & 1.692 & 1.569 & 1.842 & $<0.001$ \\
\hline Regional hospital (reference: local hospital) & 1.620 & 1.503 & 1.764 & $<0.001$ & 1.645 & 1.510 & 1.815 & $<0.001$ \\
\hline
\end{tabular}

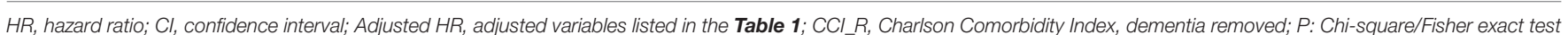
on category variables and t-test on continue variable.

with CPn pneumonia and comparison group was significant (log-rank test, $p<0.037$; Figure 1).

\section{DISCUSSION}

\section{CPn Pneumonia-AD Risk Association}

By employing a nationwide population database, our executed study is the first to demonstrate an association between CPn pneumonia and AD development in Taiwan. Patients with CPn pneumonia exhibited a nearly 1.6-fold increased risk of $\mathrm{AD}$ (overall adjusted $\mathrm{HR}=1.599 ; 95 \% \mathrm{CI}=1.284-1.971, p<0.001$ ). Additionally, hyperlipidemia, CAD, anxiety, sleep disorder, and alcohol use disorder exhibited associations with a relatively high risk of $\mathrm{AD}$. Patients who visited medical centers or regional hospitals to seek medical care were also noted to be at relatively high risk. Although the percentage of hypertension in CPn exposed cohort was higher than that in the unexposed cohort $(p<0.001)$, in Fine \& Gray's survival analysis, there were no significant differences for hypertension between the two cohorts $(p=0.087)$. However, patients who sought medical care in Autumn and with cancer revealed a reduced risk of AD. Further studies are needed to clarify why patients with these comorbidities were associated with a decreased risk. Moreover, our study also indicated that patients who were treated with macrolides ( $\geq 15$ days) and fluoroquinolones ( $\geq 15$ days) could attenuate the risk of $\mathrm{AD}$. 
TABLE 3 | Factors for Alzheimer dementia among different usages of antibiotics by using Fine and Gray's competing risk model.

\begin{tabular}{|c|c|c|c|c|c|}
\hline Medications & & Adjusted HR & $95 \% \mathrm{Cl}$ & $95 \% \mathrm{Cl}$ & $\boldsymbol{P}$ \\
\hline \multirow[t]{6}{*}{ Macrolides } & Without Chlamydia pneumoniae & Reference & & & \\
\hline & With Chlamydia pneumoniae & & & & \\
\hline & Without Macrolides & 2.177 & 1.865 & 2.468 & $<0.001$ \\
\hline & with Macrolides 1-7 days & 1.483 & 1.069 & 1.825 & 0.003 \\
\hline & with Macrolides 8-14 days & 1.592 & 1.276 & 1.899 & $<0.001$ \\
\hline & with Macrolides $\geq 15$ days & 1.198 & 0.929 & 1.487 & 0.304 \\
\hline \multirow[t]{6}{*}{ Fluoroquinolones } & Without Chlamydia pneumoniae & Reference & & & \\
\hline & With Chlamydia pneumoniae & & & & \\
\hline & Without Fluoroquinolones & 1.996 & 1.395 & 2.896 & $<0.001$ \\
\hline & With Fluoroquinolones 1-7 days & 1.506 & 1.283 & 1.883 & $<0.001$ \\
\hline & With Fluoroquinolones 8-14 days & 1.385 & 1.106 & 1.796 & 0.001 \\
\hline & With Fluoroquinolones $\geq 15$ days & 1.302 & 0.973 & 1.656 & 0.391 \\
\hline
\end{tabular}

PYS, Person-years; Adjusted HR, Adjusted variables listed in the table; Cl, confidence interval; P, Chi-square/Fisher exact test on category variables and t-test on continue variable.

Patients with Chlamydia pneumoniae pneumonia are at an increased risk of developing Alzheimer dementia

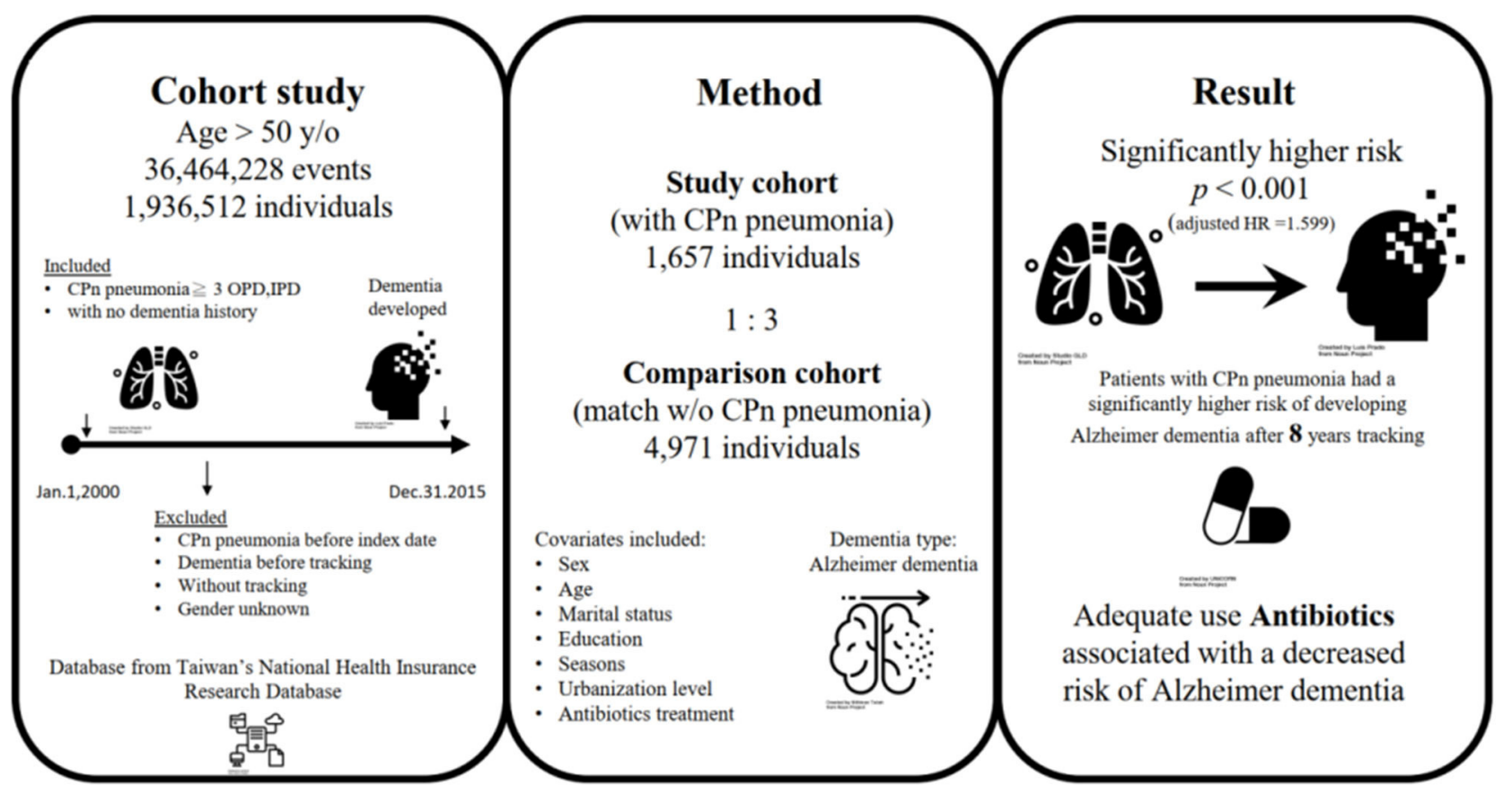

FIGURE 1 | The graphic abstract of study design and results from National Health Insurance Research Database in Taiwan. CPn, Chlamydia pneumoniae; OPD, outpatient clinic; IPD, Inpatient departments; HR, Hazard Ratio. All icons are from the Noun Project.

\section{Comparison of This Study to Previous Literatures}

Some previous studies have found that CPn was associated with AD (Balin et al., 1998; Gerard et al., 2006; Paradowski et al., 2007), but other studies had failed to detect such an association (Taylor et al., 2002; Wozniak et al., 2003; Yamamoto et al., 2005; Hammond et al., 2010). In one meta-analysis-the procedures of which entailed pooling cases and controls in previous studies-chlamydial infection was noted to exhibit an association with a 5-fold rise in the $\mathrm{AD}$ occurrence (OR: 5.66; 95\% CI $=1.83-17.51, p<0.001$ ) (Maheshwari and Eslick, 2015). In the present study, because the pneumonia diagnosis as recorded in the NHIRD was determined to have $98.3 \%$ accuracy (Su et al., 2014), we restricted patients with CPn infection to those with CPn pneumonia to bolster the credibility of the association between $\mathrm{AD}$ and $\mathrm{CPn}$. 


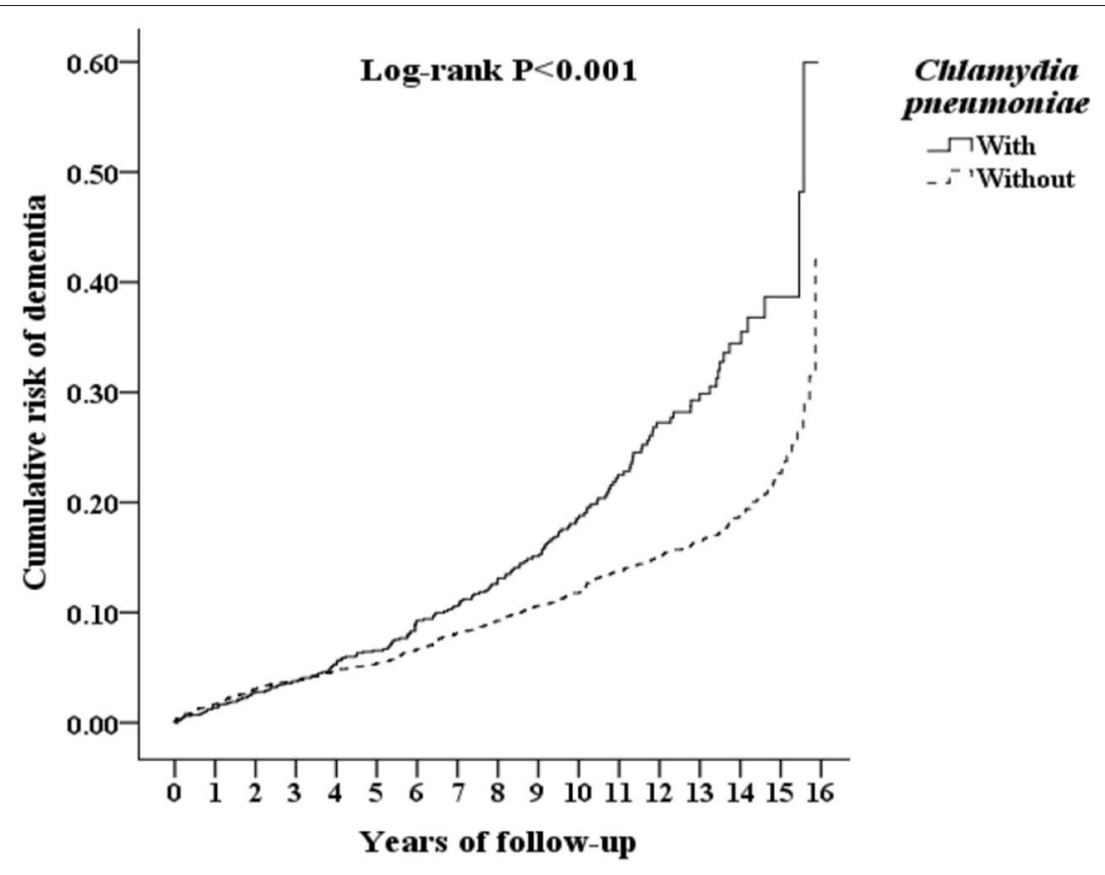

\begin{tabular}{cccc}
\hline Chlamydia pneumoniae & With $(n=1,657)$ & Without $(n=4,971)$ & P \\
\cline { 1 - 3 } In the tracking of x year(s) & \multicolumn{2}{c}{ Numbers of dementia } & \\
\hline $\mathbf{1}$ & 17 & 70 & 0.410 \\
$\mathbf{2}$ & 32 & 120 & 0.894 \\
$\mathbf{3}$ & 43 & 148 & 0.759 \\
$\mathbf{4}$ & 58 & 175 & 0.891 \\
$\mathbf{5}$ & 69 & 194 & 0.638 \\
$\mathbf{6}$ & 90 & 228 & 0.373 \\
$\mathbf{7}$ & 99 & 285 & 0.084 \\
$\mathbf{8}$ & 114 & 290 & 0.037 \\
$\mathbf{9}$ & 124 & 317 & 0.028 \\
$\mathbf{1 0}$ & 139 & 338 & 0.011 \\
$\mathbf{1 1}$ & 153 & 369 & $<0.001$ \\
$\mathbf{1 2}$ & 166 & 387 & $<0.001$ \\
$\mathbf{1 3}$ & 171 & 405 & $<0.001$ \\
$\mathbf{1 4}$ & 177 & 424 & 0.009 \\
$\mathbf{1 5}$ & 180 & 444 & $<0.001$ \\
$\mathbf{1 6}$ & 182 & 456 & $<0.001$ \\
\hline
\end{tabular}

FIGURE 2 | Kaplan-Meier for cumulative risk of dementia aged 50 and over stratified by Chlamydia pneumoniae with log-rank test.

\section{How CPn Pneumonia Might Increase the Risk of AD}

The first formal characterization of $\mathrm{AD}$ was made in 1907; nevertheless, its corresponding etiology remains poorly understood. $\mathrm{AD}$ is commonly regarded as a neurodegenerative disease and is ascribed to neuronal damage and death. Neuropathology in AD is characterized by neuropil threads, neurofibrillary tangles, neurotic senile plaques, and typically amyloid deposits around the cerebral vasculature. Neuroinflammatory processes, oxidative stress, and vascular factors are the three main contributors to $\mathrm{AD}$ pathogenesis (Ashraf et al., 2019).

Many studies have confirmed the relationship between neuroinflammation and $\mathrm{AD}$, despite the mechanistic links requiring further investigation. Herpes simplex type 1, human herpesvirus 6, Borrelia burgdorferi, and Helicobacter pylori are among the myriad of pathogens that may be involved in AD (Mawanda and Wallace, 2013). Neuroinflammation in the brain may contribute to $\mathrm{AD}$ pathogenesis. Among various pathogens, researchers have proposed CPn may cause 
chronic neuroinflammation and be implicated in AD. CPn infection of endothelial cells-infection at the vascular levelcould engender increased monocyte migration and advance inflammation in the CNS (MacIntyre et al., 2003). Moreover, in THP1 human monocytes, CPn infection stimulates an innate immune response, and thus, such infection may have a role in inflammation initiation in cases of sporadic/lateonset Alzheimer disease (Lim et al., 2014). Some animal studies have determined that $\mathrm{CPn}$ is related to beta-amyloid $(\mathrm{A} \beta) 1-$ 42 immunoreactive deposits in rodent brain tissues (Little et al., 2004; Boelen et al., 2007). A recent in vitro research claimed that CPn infection also altered the expression and activity of amyloid precursor protein and promoted amyloid formation (Al-Atrache et al., 2019). These findings provided evidence for a link between $\mathrm{CPn}$ and $\mathrm{AD}$ pathology.

According to the antimicrobial protection hypothesis (Moir et al., 2018), $A \beta$ oligomerization is not inherently pathological; $\mathrm{A} \beta$ deposition represents an early innate immune reaction to an immunochallenge, whether genuine or mistakenly perceived. $A \beta$ fibrillization inactivates neuroinflammatory pathways to defend against infection and clear the deposition of $A \beta$ or pathogens. In cases of $\mathrm{AD}$, sustained inflammation and neurodegeneration result from chronic activation of the pathway. It could be explained the cumulative incidence of $\mathrm{AD}$ in patients with $\mathrm{CPn}$ pneumonia exhibited significant after 8 years of tracking in this study.

In addition, the apolipoprotein E4 (APOE) genotype may be involved in the CPn-AD association (Wehr et al., 2006). Scholars have determined the APOE gene to be involved in cerebral $\mathrm{A} \beta$ clearance, with $\mathrm{CPn}$ influencing neuronal damage repair ability (Masters et al., 2015; Woods et al., 2020). Hence, another possible mechanism underlying CPn toxicity in $\mathrm{AD}$ may be the interaction of the APOE genotype with CPn.

As revealed in the literature, antibiotics against CPn were noted to decrease the deposition of amyloids, oxidative stress, and inflammation in AD models (Yulug et al., 2018; Balducci and Forloni, 2019); these antibiotics possess the capacity to traverse the blood-brain barrier. In this study, we noted macrolide use ( $\geq 15$ days) and fluoroquinolone use ( $\geq 15$ days) to exhibit an association with a decrease in dementia risk. Compared with macrolides, fluoroquinolones are much more lipophilic and smaller and are hence more efficacious when administered in CNS. Macrolides cannot cross the blood-brain barrier, but they are usually the first-line therapy for CPn infection; no evidence has suggested macrolides are neurotoxic or affect AD risk, and it is presumed such drugs (i.e., azithromycin, clarithromycin) do not exacerbate AD (Woods et al., 2020). The role of antibiotics against $\mathrm{CPn}$ and the risk of $\mathrm{AD}$ requires further investigation.

Pneumonia caused by CPn is typically mild, and most patients recover without complications. Our study indicated that the appropriate use of antibiotics with macrolides and fluoroquinolones (both duration $\geq 15$ days) could decrease the risk of developing $\mathrm{AD}$. In one clinical practice guideline, continue antibiotic therapy is strongly recommended in patients with CPn infection until they achieve clinical stability including resolution of vital sign abnormalities, appetite, and normal mentation (Metlay et al., 2019).
$\mathrm{CPn}$ is a type of community-acquired pneumonia and is transmitted person-to-person through inhalation of respiratory droplets or contact with droplets on surfaces followed by contact (i.e., touching) with the mouth or nose. CPn reinfection among older adults is common, and outbreaks have been reported in settings of close contact and crowding, such as nursing homes, schools, prisons, and military barracks (Burillo and Bouza, 2010). This transmissibility may explain why we noted an association between a high $\mathrm{AD}$ risk undergoing medical care at regional hospitals or medical centers.

\section{Limitations}

The NHIRD recorded inpatient care, ambulatory care, dental care, and prescription drugs availed by the insured and their date of birth. However, pursuant to the Personal Information Protection Act, individual identifiers are encrypted before releasing for research.

Therefore, information such as weakness severity, laboratory parameters, neurological symptom severity, additional examination findings (e.g., electrophysiological testing), or rehabilitation availability could not be assessed in our executed study due to the lack of such data in the NHIRD.

Besides, we could not include data on psychosocial, environmental, and genetic factors in our analyses due to the same reason. However, despite these limitations, our derived data are highly likely to be valid and representative due to the NHIRD containing data covering all hospitals within Taiwan and over $99 \%$ of the population for the relevant 15 -year period.

\section{CONCLUSIONS}

This study determined CPn pneumonia to be associated with an estimated 1.6-fold increased risk of $\mathrm{AD}$, which should alert physicians to be attentive to the risk of $\mathrm{AD}$ following $\mathrm{CPn}$ pneumonia, especially after 8 years of tracking. We noted the $\mathrm{AD}$ risk to be reduced among $\mathrm{CPn}$ pneumonia patients when administered appropriate antibiotics. We recommend the execution of additional studies based on extensive or national data sets to corroborate the present findings and elucidate the corresponding underlying mechanisms.

\section{DATA AVAILABILITY STATEMENT}

The data on the study population that were obtained from the NHIRD (http://nhird.nhri.org.tw/en/index.html) are maintained in the NHIRD (http://nhird.nhri.org.tw/). The NHRI is a nonprofit foundation established by the government. Only citizens of Taiwan who fulfill the requirements of conducting research projects are eligible to apply for access to the NHIRD. The use of the NHIRD is limited to research purposes only. Applicants must follow the Computer-Processed Personal Data Protection Law (http://www.winklerpartners.com/?p=987) and the related regulations of the National Health Insurance Administration and NHRI, and an agreement must be signed by the applicant and their supervisor upon application submission. All applications are reviewed for approval of data release. 


\section{ETHICS STATEMENT}

The studies involving human participants were reviewed and approved by The Institutional Review Board of the Tri-Service General Hospital (IRB No. 2-107-05-026). Written informed consent for participation was not required for this study in accordance with the national legislation and the institutional requirements.

\section{AUTHOR CONTRIBUTIONS}

The contributions of the authors are listed as the following: $\mathrm{HO}$ and N-ST: study concept and design. W-CC, C-HC, and N-ST: acquisition of data. W-CC, C-HC, H-AC, Y-CK, P-CW, and N-ST: analysis and interpretation of data. HO: drafting of the manuscript. N-ST: critical revision of the manuscript for important intellectual content. All authors contributed to the article and approved the submitted version.

\section{FUNDING}

This study was supported by the Tri-Service General Hospital Research Foundation under the grants of the Medical Affairs

\section{REFERENCES}

Al-Atrache, Z., Lopez, D. B., Hingley, S. T., and Appelt, D. M. (2019). Astrocytes infected with Chlamydia pneumoniae demonstrate altered expression and activity of secretases involved in the generation of $\beta$-amyloid found in Alzheimer disease. BMC Neurosci. 20:6. doi: 10.1186/s12868-019-0489-5

Ashraf, G. M., Tarasov, V. V., Makhmutov, A., Chubarev, V. N., AvilaRodriguez, M., Bachurin, S. O., et al. (2019). The possibility of an infectious etiology of Alzheimer disease. Mol. Neurobiol. 56, 4479-4491. doi: $10.1007 /$ s12035-018-1388-y

Balducci, C., and Forloni, G. (2019). Doxycycline for Alzheimer's disease: fighting $\beta$-amyloid oligomers and neuroinflammation. Front. Pharmacol. 10:738. doi: 10.3389/fphar.2019.00738

Balin, B. J., Gerard, H. C., Arking, E. J., Appelt, D. M., Branigan, P. J., Abrams, J. T., et al. (1998). Identification and localization of Chlamydia pneumoniae in the Alzheimer's brain. Med. Microbiol. Immunol. 187, 23-42. doi: $10.1007 / \mathrm{s} 004300050071$

Balin, B. J., Hammond, C. J., Little, C. S., Hingley, S. T., Al-Atrache, Z., Appelt, D. M., et al. (2018). Chlamydia pneumoniae: an etiologic agent for late-onset dementia. Front. Aging Neurosci. 10:302. doi: 10.3389/fnagi.2018.00302

Bloom, G. S. (2014). Amyloid-beta and tau: the trigger and bullet in Alzheimer disease pathogenesis. JAMA Neurol. 71, 505-508. doi: 10.1001/jamaneurol.2013.5847

Boelen, E., Stassen, F. R., van der Ven, A. J., Lemmens, M. A., Steinbusch, H. P., Bruggeman, C. A., et al. (2007). Detection of amyloid beta aggregates in the brain of $\mathrm{BALB} / \mathrm{c}$ mice after Chlamydia pneumoniae infection. Acta Neuropathol. 114, 255-261. doi: 10.1007/s00401-007-0252-3

Burillo, A., and Bouza, E. (2010). Chlamydophila pneumoniae. Infect. Dis. Clin. North Am. 24, 61-71. doi: 10.1016/j.idc.2009.10.002

Chang, C. Y., Chen, W. L., Liou, Y. F., Ke, C. C., Lee, H. C., Huang, H. L., et al. (2014). Increased risk of major depression in the three years following a femoral neck fracture-a national population-based follow-up study. PLoS ONE 9, e89867. doi: 10.1371/journal.pone.0089867

Chang, S. Y., Chien, W. C., Chung, C. H., Chang, H. A., Kao, Y. C., Yeh, H. W., et al. (2018). The risk of dementia after charcoal-burning suicide attemptsa nationwide cohort study in Taiwan. J. Investig. Med. 66, 1070-1082. doi: 10.1136/jim-2018-000759
Bureau, the Ministry of Defense of Taiwan (MAB-107-084 and MND-MAB-110-087), the Tri-Service General Hospital Research Foundation (TSGH-C108-151, TSGH-E-110240, and TSGH-B110-012), and the Taoyuan Armed Forces General Hospital (TYAFGH-A-110020). The sponsor had no role in the study design, data collection and analysis, decision to publish, or preparation of the manuscript.

\section{ACKNOWLEDGMENTS}

We appreciate the provision of the National Health Insurance Research Database by the Taiwan's Health and Welfare Data Science Center and Ministry of Health and Welfare (HWDC, MOW). Icons from Figure 2 were attributed to neurodegenerative by Nithinan Tatah, Alzheimer's Disease by Luis Prado, Pneumonia by Studio GLD, Antibiotics by UNiCORN, Data by ic2icon. All icons are from the Noun Project.

\section{SUPPLEMENTARY MATERIAL}

The Supplementary Material for this article can be found online at: https://www.frontiersin.org/articles/10.3389/fnagi. 2021.701899/full\#supplementary-material

Chao, P. C., Chien, W. C., Chung, C. H., Chu, C. W., Yeh, C. B., Huang, S. Y., et al. (2018). Cognitive enhancers associated with decreased risk of injury in patients with dementia: a nationwide cohort study in Taiwan. J. Investig. Med. 66, 684-692. doi: 10.1136/jim-2017000595

Gerard, H. C., Dreses-Werringloer, U., Wildt, K. S., Deka, S., Oszust, C., Balin, B. J., et al. (2006). Chlamydophila (Chlamydia) pneumoniae in the Alzheimer's brain. FEMS Immunol. Med. Microbiol. 48, 355-366. doi: 10.1111/j.1574-695X.2006.00154.x

Gottesman, R. F., Albert, M. S., Alonso, A., Coker, L. H., Coresh, J., Davis, S. M., et al. (2017). Associations between midlife vascular risk factors and 25-year incident dementia in the atherosclerosis risk in communities (ARIC) cohort. JAMA Neurol. 74, 1246-1254. doi: 10.1001/jamaneurol.201 7.1658

Hammond, C. J., Hallock, L. R., Howanski, R. J., Appelt, D. M., Little, C. S., and Balin, B. J. (2010). Immunohistological detection of Chlamydia pneumoniae in the Alzheimer's disease brain. BMC Neurosci. 11:121. doi: 10.1186/1471-2202-11-121

Lim, C., Hammond, C. J., Hingley, S. T., and Balin, B. J. (2014). Chlamydia pneumoniae infection of monocytes in vitro stimulates innate and adaptive immune responses relevant to those in Alzheimer's disease. J. Neuroinflammation 11, 1-11. doi: 10.1186/s12974-014-0217-0

Little, C. S., Hammond, C. J., MacIntyre, A., Balin, B. J., and Appelt, D. M. (2004). Chlamydia pneumoniae induces Alzheimer-like amyloid plaques in brains of BALB/c mice. Neurobiol. Aging 25, 419-429. doi: 10.1016/S0197-4580(03)00127-1

Loeb, M. B., Molloy, D. W., Smieja, M., Standish, T., Goldsmith, C. H., Mahony, J., et al. (2004). A randomized, controlled trial of doxycycline and rifampin for patients with Alzheimer's disease. J. Am. Geriatr. Soc. 52, 381-387. doi: 10.1111/j.1532-5415.2004.52109.x

MacIntyre, A., Abramov, R., Hammond, C. J., Hudson, A. P., Arking, E. J., Little, C. S., et al. (2003). Chlamydia pneumoniae infection promotes the transmigration of monocytes through human brain endothelial cells. J. Neurosci. Res. 71, 740-750. doi: 10.1002/jnr.10519

Maheshwari, P., and Eslick, G. D. (2015). Bacterial infection and Alzheimer's disease: a meta-analysis. J. Alzheimers. Dis. 43, 957-966. doi: 10.3233/JAD-140621 
Masters, C. L., Bateman, R., Blennow, K., Rowe, C. C., Sperling, R. A., and Cummings, J. L. (2015). Alzheimer's disease. Nat. Rev. Dis. Primers 433. 1:434. doi: $10.1038 / \mathrm{nrdp} .2015 .56$

Mawanda, F., and Wallace, R. (2013). Can infections cause Alzheimer's disease? J. Epidemiol. Rev. 35, 161-180. doi: 10.1093/epirev/mxs007

Metlay, J. P., Waterer, G. W., Long, A. C., Anzueto, A., Brozek, J., Crothers, K., et al. (2019). Diagnosis and treatment of adults with community-acquired pneumonia. an official clinical practice guideline of the American Thoracic Society and Infectious Diseases Society of America. Am. J. Respir. Crit. Care Med. 200, e45-e67. doi: 10.1164/rccm.2019081581ST

Moir, R. D., Lathe, R., and Tanzi, R. E. (2018). The antimicrobial protection hypothesis of Alzheimer's disease. Alzheimer's Dementia 14, 1602-1614. doi: $10.1016 /$ j.jalz.2018.06.3040

National Health Insurance Administration National Health Insurance Regulations [Online]. Available online at: http://www.who.int/mediacentre/news/releases/ 2012/dementia_20120411/en/index.htm (accessed June 23, 2016).

Needham, D. M., Scales, D. C., Laupacis, A., and Pronovost, P. J. (2005). A systematic review of the Charlson comorbidity index using Canadian administrative databases: a perspective on risk adjustment in critical care research. J. Crit. Care 20, 12-19. doi: 10.1016/j.jcrc.2004.09.007

Paradowski, B., Jaremko, M., Dobosz, T., Leszek, J., and Noga, L. (2007). Evaluation of CSF-Chlamydia pneumoniae, CSF-tau, and CSF-Abeta42 in Alzheimer's disease and vascular dementia. J. Neurol. 254, 154-159. doi: 10.1007/s00415-006-0298-5

Su, V. Y., Liu, C. J., Wang, H. K., Wu, L. A., Chang, S. C., Perng, D. W., et al. (2014). Sleep apnea and risk of pneumonia: a nationwide population-based study. CMAJ 186, 415-421. doi: 10.1503/cmaj.131547

Sun, Y., Lee, H. J., Yang, S. C., Chen, T. F., Lin, K. N., Lin, C. C., et al. (2014). A nationwide survey of mild cognitive impairment and dementia, including very mild dementia, in Taiwan. PLoS ONE 9, e100303. doi: 10.1371/journal.pone.0100303

Taylor, G. S., Vipond, I. B., Paul, I. D., Matthews, S., Wilcock, G. K., and Caul, E. O. (2002). Failure to correlate C. pneumoniae with late onset Alzheimer's disease. Neurology 59, 142-143. doi: 10.1212/WNL.59.1.142

Tzeng, N. S., Chang, C. W., Hsu, J. Y., Chou, Y. C., Chang, H. A., and Kao, Y. C. (2015). Caregiver burden for patients with dementia with or without hiring foreign health aides: a cross-sectional study in a northern taiwan memory clinic. J. Med. Sci. 35, 239-247. doi: 10.4103/1011-4564.1 72999

van den Berg, B., Bunschoten, C., van Doorn, P. A., and Jacobs, B. C. (2013). Mortality in Guillain-Barré syndrome. Neurology 80, 1650-1654. doi: 10.1212/WNL.0b013e3182904fcc

van den Berg, B., Walgaard, C., Drenthen, J., Fokke, C., Jacobs, B. C., and van Doorn, P. A. (2014). Guillain-Barre syndrome: pathogenesis, diagnosis, treatment and prognosis. Nat. Rev. Neurol. 10, 469-482. doi: 10.1038/nrneurol.2014.121

Wehr, H., Bednarska-Makaruk, M., Łojkowska, W., Graban, A., HoffmanZacharska, D., Kuczyńska-Zardzewiały, A., et al. (2006). Differences in risk factors for dementia with neurodegenerative traits and for vascular dementia. Dement. Geriatr. Cogn. Disord. 22, 1-7. doi: 10.1159/000092845

Wong, A. H. Y., Umapathi, T., Shahrizaila, N., Chan, Y. C., Kokubun, N., Fong, M. K., et al. (2014). The value of comparing mortality of Guillain-Barré syndrome across different regions. J. Neurol. Sci. 344, 60-62. doi: 10.1016/j.jns.2014.06.021

Woods, J. J., Skelding, K. A., Martin, K. L., Aryal, R., Sontag, E., Johnstone, D. M., et al. (2020). Assessment of evidence for or against contributions of Chlamydia pneumoniae infections to Alzheimer's disease etiology. Brain Behav. Immun. 83, 22-32. doi: 10.1016/j.bbi.2019.10.014

Wozniak, M. A., Cookson, A., Wilcock, G. K., and Itzhaki, R. F. (2003). Absence of Chlamydia pneumoniae in brain of vascular dementia patients. Neurobiol. Aging 24, 761-765. doi: 10.1016/S0197-4580(02)00236-1

Wright, C. B., Gardener, H., Dong, C., Yoshita, M., DeCarli, C., Sacco, R. L., et al. (2015). Infectious burden and cognitive decline in the northern Manhattan study. J. Am. Geriatr. Soc. 63, 1540-1545. doi: 10.1111/jgs.13557

Yamamoto, H., Watanabe, T., Miyazaki, A., Katagiri, T., Idei, T., Iguchi, T., et al. (2005). High prevalence of Chlamydia pneumoniae antibodies and increased high-sensitive C-reactive protein in patients with vascular dementia. J. Am. Geriatr. Soc. 53, 583-589. doi: 10.1111/j.1532-5415.2005.53204.x

Yulug, B., Hanoglu, L., Ozansoy, M., Isik, D., Kilic, U., Kilic, E., et al. (2018). Therapeutic role of rifampicin in Alzheimer's disease. Psychiatry Clin. Neurosci. 72, 152-159. doi: $10.1111 /$ pcn.12637

Conflict of Interest: The authors declare that the research was conducted in the absence of any commercial or financial relationships that could be construed as a potential conflict of interest.

Publisher's Note: All claims expressed in this article are solely those of the authors and do not necessarily represent those of their affiliated organizations, or those of the publisher, the editors and the reviewers. Any product that may be evaluated in this article, or claim that may be made by its manufacturer, is not guaranteed or endorsed by the publisher.

Copyright (c) 2021 Ou, Chien, Chung, Chang, Kao, Wu and Tzeng. This is an openaccess article distributed under the terms of the Creative Commons Attribution License (CC BY). The use, distribution or reproduction in other forums is permitted, provided the original author(s) and the copyright owner(s) are credited and that the original publication in this journal is cited, in accordance with accepted academic practice. No use, distribution or reproduction is permitted which does not comply with these terms. 